\title{
Bone mineral density, body mass index and cigarette smoking among Iranian women: implications for prevention Azam Baheiraei ${ }^{1}$, Nicholas A Pocock ${ }^{2}$, John A Eisman ${ }^{1}$, Nguyen D Nguyen ${ }^{1}$ and Tuan V Nguyen*1
}

\author{
Address: 'Bone and Mineral Research Program, Garvan Institute of Medical Research, St Vincent's Hospital, University of New South Wales, \\ Sydney, Australia and 2Department of Nuclear Medicine, St Vincent's Hospital, Sydney, Australia \\ Email: Azam Baheiraei - a.baheiraei@garvan.org.au; Nicholas A Pocock - n.pocock@unsw.edu.au; John A Eisman - j.eisman@garvan.org.au; \\ Nguyen D Nguyen - n.nguyen@garvan.org.au; Tuan V Nguyen* - t.nguyen@garvan.org.au \\ * Corresponding author
}

Published: 24 June 2005

BMC Musculoskeletal Disorders 2005, 6:34 doi:10.1 186/147/-2474-6-34

This article is available from: http://www.biomedcentral.com/I47|-2474/6/34

(c) 2005 Baheiraei et al; licensee BioMed Central Ltd.

This is an Open Access article distributed under the terms of the Creative Commons Attribution License (http://creativecommons.org/licenses/by/2.0), which permits unrestricted use, distribution, and reproduction in any medium, provided the original work is properly cited.
Received: 15 December 2004

Accepted: 24 June 2005

\begin{abstract}
Background: While risk factors of osteoporosis in Western populations have been extensively documented, such a profile has not been well studied in Caucasians of non-European origin. This study was designed to estimate the modifiable distribution and determinants of bone mineral density (BMD) among Iranian women in Australia.
\end{abstract}

Methods: Ninety women aged 35 years and older completed a questionnaire on sociodemographic and lifestyle factors. BMD was measured at the lumbar spine (LS) and femoral neck (FN) using DXA (GE Lunar, WI, USA), and was expressed in $\mathrm{g} / \mathrm{cm}^{2}$ as well as T-score.

Results: In multiple regression analysis, advancing age, lower body mass index (BMI), and smoking were independently associated with LS and FN BMD, with the 3 factors collectively accounting for $30 \%$ and $38 \%$ variance of LS and FN BMD, respectively. LS and FN BMD in smokers was $8 \%$ lower than that in non-smokers. Further analysis of interaction between BMI and smoking revealed that the effect of smoking was only observed in the obese group $(p=0.029$ for LSBMD and $p=0.007$ for FNBMD), but not in the overweight and normal groups. Using T-scores from two bone sites the prevalence of osteoporosis (T-scores $\leq-2.5$ ) was $3.8 \%$ and $26.3 \%$ in pre-and post-menopausal women, respectively. Among current smokers, the prevalence was higher (3I.3\%) than that among ex-smokers (28.6\%) and non-smokers (7.5\%).

Conclusion: These data, for the first time, indicate that apart from advancing age and lower body mass index, cigarette smoking is an important modifiable determinant of bone mineral density in these Caucasians of non-European origin.

\section{Background}

Osteoporosis is a common disorder in the elderly population, and represents one of the most significant public health problems in the world, predisposing to fractures with minimal or no antecedent trauma. These fractures are, in turn, associated with increased morbidity [1], reduced quality of life [2], mortality [3], and high health care costs [4]. 
Bone mineral density (BMD) measurement is considered an effective predictor of fracture risk, such that each standard deviation lower in BMD is associated with at least a 2fold increase in age adjusted fracture risk. [5-7]. Therefore, a useful approach in assessing the importance of aetiological factors for osteoporosis is an investigation of the distribution and determinants of BMD. Although determinants of bone mineral density in Western populations have been extensively studied, such a profile has not been well documented in Caucasians of non-European origin.

Body weight or body mass index (BMI) is known to be positively associated with BMD[8,9]. Lifestyle factors such as low calcium intake, lack of physical activity, and smoking adversely affect bone mineral density and increase the risk of osteoporosis and its related fractures[10]. These factors also play an important role in the determination of peak bone mass and subsequent bone loss during the post-menopausal period. Among the modifiable risk factors of osteoporosis, cigarette smoking is considered one of the deleterious factors because cigarette smokers also have increased risk of fracture $[11,12]$. Nevertheless, the interactive effect of smoking on BMD has not been well studied. A recent study in a Caucasian population suggested that the effect of smoking was modified by body mass index, such that non-obese smokers had lower BMD than obese-smokers[13]. Iranian women on the average have a relatively high $\mathrm{BMI}[14,15]$, and it is not known whether such an interaction effect between smoking and $\mathrm{BMI}$ is present in this population.

The present study was designed to examine the modifiable distribution and determinants of bone mineral density among Iranian Australian women.

\section{Methods}

\section{Subjects and setting}

This study was designed as a cross-sectional investigation. All women were recruited via a media campaign using newsletters, noticeboards in community halls as well as word of mouth at community centres as part of a larger study to examine osteoporosis prevention in Iranian women. Inclusion criteria for the study were Iranian women and aged 35 years or older. The exclusion criteria were: current or past occurrence of any medical conditions known to affect bone metabolism such as Paget's disease and stroke; current pregnancy; and/or a history of breastfeeding within the last year. Also excluded were women who had been taking any medication affecting bone such as hormones, calcium, and glucocorticoids. In total, 96 women participated in the current study. Six women, who did not meet study's criteria on the basis of diseases or history of taking medications affecting bone, were excluded from the analysis. This study was approved by the Univer- sity of New South Wales's Human Research Ethics Committee and written informed consent was obtained from each participant.

\section{Data collection and measurements}

\section{Socio-demographic characteristics and lifestyle risk factors}

Each woman completed a modified structured questionnaire [16] on socio-demographic and lifestyle risk factors. Income was included to be assessed, however, most participants refused to obtain information about their income level. Reproductive factors such as menopausal status and years since menopause were also provided for each participant. Menopause was defined as previous natural or surgical cessation of menstruation for more than 12 months. Calcium intake was calculated as the sum of current intake of main dairy products (milk, yogurt, and cheese) and was then converted to milligrams of calcium per day. Calcium contents for dairy products were provided from the product information in Australia [17]. Exercise was dichotomized as "yes" for current regular exercising, or "no" for not exercising. Amongst those who exercised, total amount of time spent per week was recorded. Current alcohol use was recorded as "yes" for drinking alcohol (beer, wine and liquor), or "no" for no intake of alcohol. Smoking habits were assessed based on previous and current cigarette smoking. Smoking status was dichotomized as "yes" for smoking, or "no" for never smoking. In addition, amongst those who smoked, dose and duration of smoking was recorded.

\section{Anthropometric data}

Weight $(\mathrm{kg})$ and height $(\mathrm{cm})$ were measured with light indoor clothing without shoes at the time of bone densitometry measurements. Weight was recorded to the nearest tenth of a kg using an electronic scale and standing height was measured to the nearest centimeter with a stadiometer. Body mass index (BMI) was calculated as body weight in kilograms divided by height in meters squared. According to the World Health Organization (WHO) recommended classification system, overweight and obese individuals were classified as having a BMI between 25 and 29 , and equal to or greater than $30 \mathrm{~kg} / \mathrm{m}^{2}$, respectively[18].

\section{Bone density measurement}

BMD was measured at the lumbar spine (LS) (L2-L4, anterior-posterior position) and femoral neck (FN) using dual-energy X-ray absorptiometry (DXA) with a Lunar Prodigy densitometer (GE Lunar, WI, U.S.A.). Areal BMD was expressed in $\mathrm{g} / \mathrm{cm}^{2}$ and in standard deviations from the young normal mean (T-score), based on the Australian Reference Population. The sample of women was grouped into 3 groups based on the WHO recommended criteria: osteoporosis if T-score $\leq-2.5$; osteopenia if $-2.5<$ T-score $\leq-1.0$; and normal if T-score >-1.0 [19]. 


\section{Data analysis}

To determine the magnitude of association between the potential risk factors (e.g., menopausal status, height, weight, dairy calcium intake, smoking, exercise, and alcohol use) and osteoporosis risk. Bone mineral density was considered the primary outcome, and was treated as a continuous variable. Individual risk factors were first considered in a simple linear regression analysis to estimate the strength of association between individual risk factor and BMD. In the subsequent analysis, all risk factors were simultaneously considered in a multiple linear regression analysis using the backward elimination algorithms, to screen for independent significant factors. Residual analysis performed to ensure that the usual assumptions of the regression model (i.e. normality, homogeneity and independence) were met. The entry of significance level ( $p$ value) was set to 0.10 to arrive at the most robust model.

In further analysis, differences between the pre-menopausal and post-menopausal groups were tested by unpaired t-test for the normally distributed variables, or the MannWhitney U test for non-normally distributed variables, and Chi-square test for categorized data. The analysis was performed with the SAS statistical analysis system[20] and SPSS for Windows statistical software [21].

\section{Results \\ Characteristics of study subjects}

The study population consisted of 90 women aged $48.5 \pm$ $8.3 \mathrm{yr}$ (mean age \pm SD; range: 35 to $77 \mathrm{yr}$ ). Approximately $42 \%$ of the women had education within high school. The majority of the women were married (78\%) and performing home duties or not employed (56\%). Their average duration of residence in Australia was about 10 years, with $75 \%$ of subjects having resided in Australia for at least 5 years. The mean age (SD) at immigration was about $39 \pm 9.4 \mathrm{yr}$ (range: 18 to $65 \mathrm{yr}$ ). The median (SD) dairy calcium intake in the women was $407 \pm 283 \mathrm{mg} /$ day. The Twenty-three women (26\%) exercised regularly. Approximately $26 \%$ of women smoked cigarettes during their lifetime. Although cigarette smoking was common in these subjects, alcohol use was not frequent with about $11 \%$ of the women reporting drinking any kind of alcohol. Using the BMI criteria, 2.2\% of subjects were underweight; $25.6 \%$ of women were in the healthy weight range; $35.6 \%$ were over-weight; and $36.7 \%$ were obese.

Forty two percent $(n=38)$ of women were post-menopausal, with the duration of post-menopause being between 1 and 32 years. Post-menopausal women had significantly higher age and parity and lower height, lumbar spine and femoral neck BMD, but no significant differences were found between the pre-and post-menopausal women in weight, BMI, dairy calcium intake, exercise, smoking status, duration of smoking, and alcohol use (Table 1).

Table I: Clinico-demographic characteristic of study subjects

\begin{tabular}{|c|c|c|c|}
\hline & Pre-menopause & Post-menopause & p value \\
\hline $\mathrm{N}$ & 52 & 38 & \\
\hline Age (years)* & $43.6 \pm 4.7$ & $55.18 \pm 7.4$ & $<\left.0.00\right|^{a}$ \\
\hline Height $(\mathrm{cm})^{*}$ & $157.7 \pm 5.5$ & $155 \pm 5.7$ & $0.027^{a}$ \\
\hline Weight $(\mathrm{kg})^{*}$ & $70.8 \pm 16.4$ & $68.9 \pm 10.3$ & $0.530^{a}$ \\
\hline BMI $\left(\mathrm{kg} / \mathrm{m}^{2}\right)^{*}$ & $28.5 \pm 6.9$ & $28.7 \pm 4.3$ & $0.869 a$ \\
\hline LSBMD $\left(\mathrm{g} / \mathrm{cm}^{2}\right)^{*}$ & $1.19 \pm 0.15$ & $1.04 \pm 0.16$ & $<\left.0.00\right|^{a}$ \\
\hline FNBMD $\left(\mathrm{g} / \mathrm{cm}^{2}\right)^{*}$ & $0.97 \pm 0.12$ & $0.87 \pm 0.11$ & $<\left.0.00\right|^{a}$ \\
\hline Dairy calcium intake $(\mathrm{mg} / \text { day })^{*}$ & $410 \pm 262$ & $498 \pm 306$ & $0.147^{a}$ \\
\hline Age at menopause* & - & $47.9 \pm 4.02$ & - \\
\hline Parity ${ }^{\prime}$ & $2(2,3)$ & $3(2,4)$ & $0.004^{b}$ \\
\hline Regular exercise§ & $12(23.1)$ & II (28.9) & $0.528 \mathrm{c}$ \\
\hline \multicolumn{4}{|l|}{ Smoking status } \\
\hline Current smokers & I5.4(8) & $21.1(8)$ & $0.487^{c}$ \\
\hline Ex-smokers & $21.2(\mathrm{II})$ & $23.7(9)$ & $0.775^{c}$ \\
\hline Duration of smoking (years)§ & & & $0.155^{c}$ \\
\hline$\leq 5$ & $4(33.3)$ & I (9.1) & \\
\hline$>5$ & $8(66.7)$ & $10(90.9)$ & \\
\hline Alcohol use $\S$ & $7(13.5)$ & $3(7.9)$ & $0.407^{c}$ \\
\hline
\end{tabular}

*Mean $\pm \mathrm{SD}$; tmedian (interquartile range); § $\mathrm{n}(\%)$.

aunpaired t-test, bMann-Whitney $U$ test, ${ }^{c}$ Chi-square test.

BMI, body mass index; LSBMD, lumbar spine bone mineral density; FNBMD, femoral neck bone mineral density. 
Table 2: Univariate association between individual risk factors and bone mineral density

\begin{tabular}{|c|c|c|c|c|}
\hline \multirow[t]{2}{*}{ Factor } & \multicolumn{2}{|c|}{ LSBMD $\left(\mathrm{g} / \mathrm{cm}^{2}\right)$} & \multicolumn{2}{|c|}{ FNBMD $\left(\mathrm{g} / \mathrm{cm}^{2}\right)$} \\
\hline & $\beta \pm S E$ a & $R^{2 b}$ & $\beta \pm \mathrm{SE}^{\mathrm{a}}$ & $R^{2 b}$ \\
\hline Age (per I year) & $-0.009 \pm 0.002^{*}$ & 0.20 & $-0.008 \pm 0.00 I^{*}$ & 0.25 \\
\hline Height (per $-5 \mathrm{~cm}$ ) & $0.04 \pm 0.012 *$ & 0.07 & $0.030 \pm 0.010^{*}$ & 0.07 \\
\hline Weight (per -5 kg) & $0.02 \pm 0.005^{*}$ & 0.10 & $0.015 \pm 0.005^{*}$ & 0.14 \\
\hline BMI (per -5 kg/m²) & $0.035 \pm 0.015^{*}$ & 0.05 & $0.030 \pm 0.010^{*}$ & 0.07 \\
\hline Post-menopause & $-0.153 \pm 0.033^{*}$ & 0.19 & $-0.103 \pm 0.026 *$ & 0.15 \\
\hline Smoking (current and ex-smokers) & $-0.100 \pm 0.040 *$ & 0.06 & $-0.086 \pm 0.030 *$ & 0.08 \\
\hline Duration of smoking (per 5 years) & $-0.037 \pm 0.015^{*}$ & 0.06 & $-0.031 \pm 0.011^{*}$ & 0.07 \\
\hline Cigarette dose (per 10 cig/day) & $-0.054 \pm 0.019 *$ & 0.08 & $-0.038 \pm 0.014^{*}$ & 0.07 \\
\hline Dairy calcium intake (per $300 \mathrm{mg} /$ day) & $0.020 \pm 0.03$ & 0.001 & $0.020 \pm 0.03$ & 0.02 \\
\hline Regular exercise (yes) & $0.017 \pm 0.042$ & 0.002 & $0.044 \pm 0.031$ & 0.02 \\
\hline Alcohol use (yes) & $0.004 \pm 0.058$ & 0.001 & $-0.021 \pm 0.044$ & 0.003 \\
\hline
\end{tabular}

BMI, body mass index; LSBMD, lumbar spine bone mineral density; FNBMD, femoral neck bone mineral density.

$* \mathrm{P}<0.05$ (statistically significant)

a Values are regression coefficients \pm SE describing the change in bone mineral density $\left(\mathrm{g} / \mathrm{cm}^{2}\right)$ associated with the unit change in the risk factor

b Coefficient of determination: the proportion of variation in bone mineral density explained by the variation in a risk factor

Table 3: Association between age, body mass index, smoking, and bone mineral density:Results of multiple linear regression analysis

\begin{tabular}{lll}
\hline Determinant & LSBMD $\left(\mathbf{g} / \mathrm{cm}^{2}\right)^{\mathbf{a}}$ & FNBMD $\left(\mathbf{g} / \mathrm{cm}^{2}\right)^{\mathbf{a}}$ \\
\hline Age (per I year) & $-0.008 \pm 0.002^{* *}$ & $-0.007 \pm 0.00 I^{* *}$ \\
BMI (-5 kg/m $)$ & $0.006 \pm 0.003^{*}$ & $0.005 \pm 0.002^{* *}$ \\
Smoking (yes) & $-0.087 \pm 0.035^{*}$ & $-0.075 \pm 0.025^{* *}$ \\
$\mathrm{R}^{2 *}$ & 0.30 & 0.38
\end{tabular}

$* 0.01<p<0.05 ; * * 0.0001<p<0.01$

a Values are regression coefficients \pm SE describing the change in BMD $\left(\mathrm{g} / \mathrm{cm}^{2}\right)$ associated with one year advancing age, $5 \mathrm{~kg} / \mathrm{m}^{2}$ decrease in $\mathrm{BMI}$ and smoking status.

BMI, body mass index; LSBMD, lumbar spine bone mineral density; FNBMD, femoral neck bone mineral density.

$* \mathrm{R}^{2}$, coefficient of determination: a measure of the proportion of variation in BMD explained by the variation in the risk factors. The variables included in the initial regression analysis were: age, menopause status, BMI, smoking status, duration of smoking, cigarette dose, calcium intake, exercise, and alcohol use

\section{Determinants of BMD}

In simple linear regression analysis, age, height, weight, BMI, menopausal status, smoking habits, duration of smoking, and cigarette dose were each significantly associated with LS and FN BMD (Table 2). However, in the multiple linear regression, advancing age, lower BMI and smoking were independent predictors of LS and FN BMD (Table 3). After adjusting for age and BMI, smokers had $0.087 \mathrm{~g} / \mathrm{cm}^{2}(8 \%)$ and $0.075 \mathrm{~g} / \mathrm{cm}^{2}(8 \%)$ lower in LS and FN BMD, respectively, than non-smokers. The 3 factors collectively accounted for $30 \%$ and $38 \%$ of the variation in LS and FN BMD, respectively.
As expected, advancing age was negatively associated with BMD in the both sites (LS: $r=0.45, p=0.0001$; FN: $r=$ $0.50, p=0.0001)$. Nevertheless, there was a significant positive correlation between BMI and LS and FN BMD (LS: $r=0.22, p=0.033$; FN: $r=0.26, p=0.012$ ). Current smokers had significantly lower lumbar spine and femoral neck BMD than non-smokers. However, there was no significant difference between ex-smokers and non-smokers in both BMD sites (Fig. 1). Among smokers, there was no significant linear correlation between cigarette dose and BMD ( $p=0.14$ for LSBMD and $p=0.64$ for FNBMD) and duration of smoking and BMD ( $p=0.76$ for LSBMD and $p=0.86$ for FNBMD).

Further analysis of interaction between BMI and smoking revealed that the effect of smoking was only observed in the obese group ( $p=0.029$ for LSBMD and $p=0.007$ for FNBMD), but not in the overweight and normal groups (Fig. 2). This interaction effect was not affected by the dose of cigarette or duration of smoking. Moreover, there was a non-statistically significant interaction between age and smoking, as both smokers and non-smokers appeared to have a similar age-BMD association (Fig. 3).

\section{Prevalence of low bone density}

Twenty-five women (27.8\%) were osteopenic (T-score -1 to -2.49) at the lumbar spine and 32 (35.6\%) at the femoral neck. Using the WHO T-score-based definition of osteoporosis, the proportion of women with osteoporosis was $12.2 \%(n=11)$ at the lumbar spine and $2.2 \%(n=2)$ at the femoral neck. When the two measures were considered simultaneously, the prevalence of osteoporosis was $13.3 \%$. In post-menopausal women, the prevalence of osteoporosis (T-score $\leq-2.5)$ was $23.7 \%(n=9)$ at the 

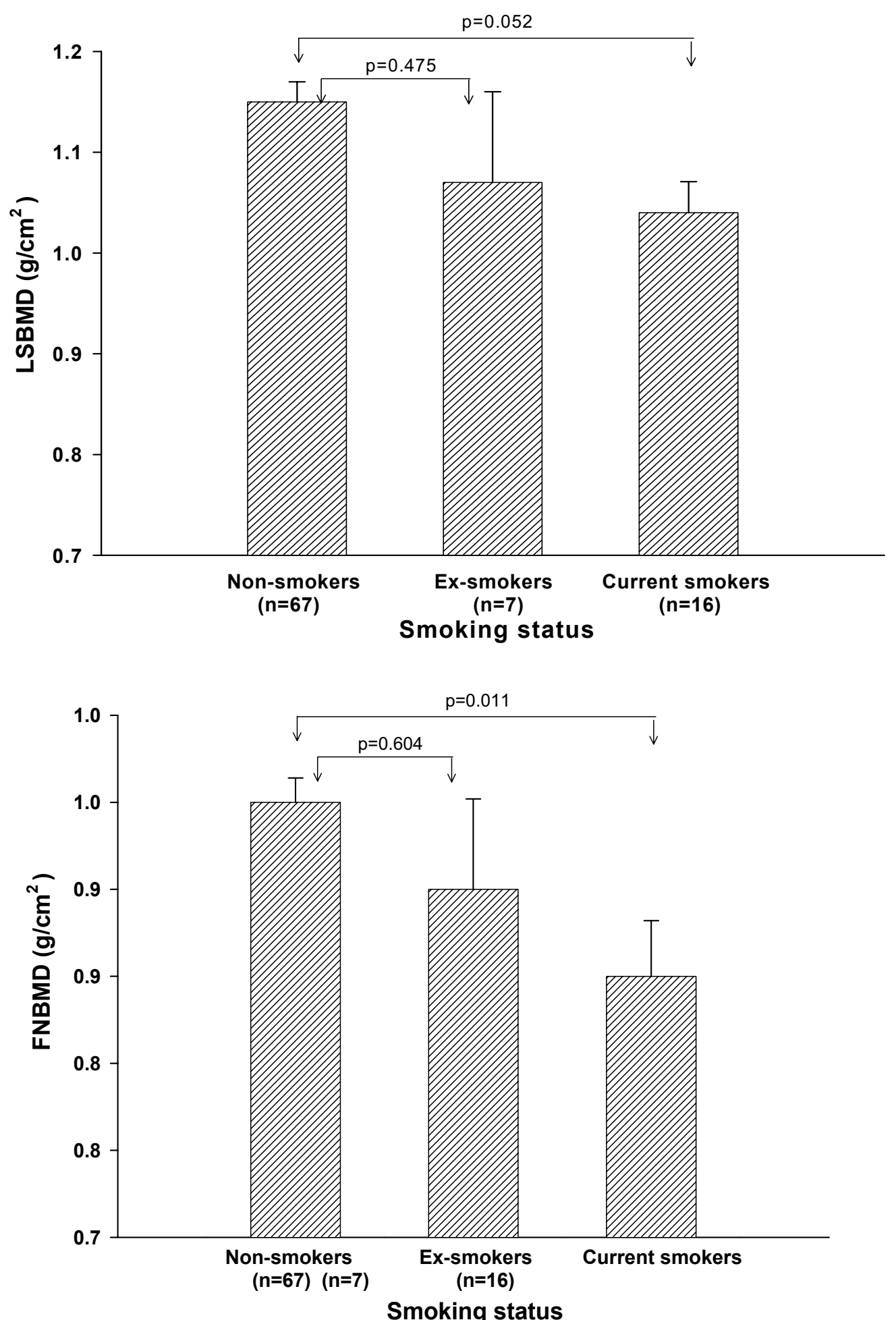

Figure I

Mean and standard error of lumbar spine (upper panel) and femoral neck (lower panel) bone mineral density (g/cm²) by smoking status. 

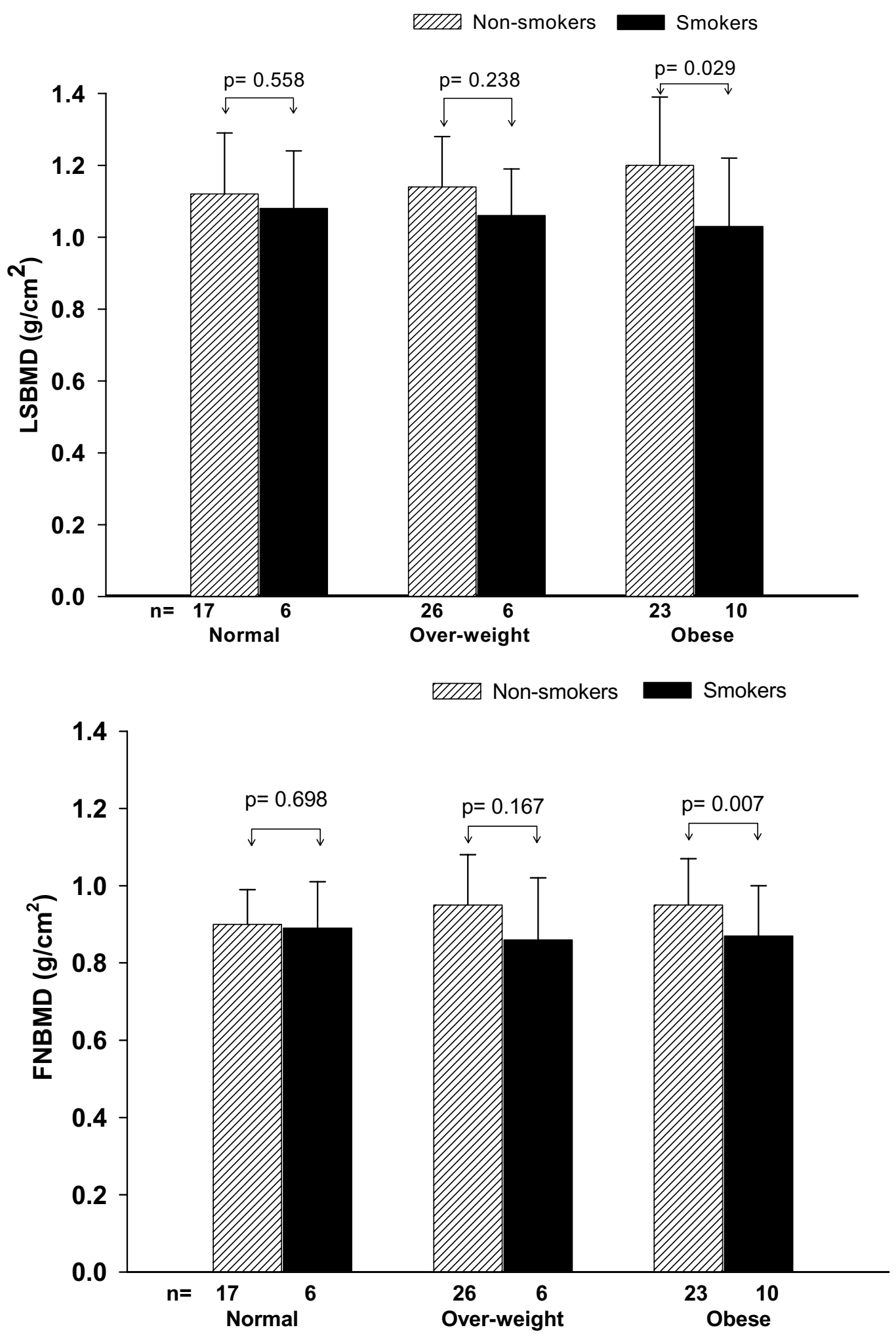

Figure 2

Mean and standard error of lumbar spine (upper panel) and femoral neck (lower panel) bone mineral density (g/cm²) by body mass index and smoking status. 

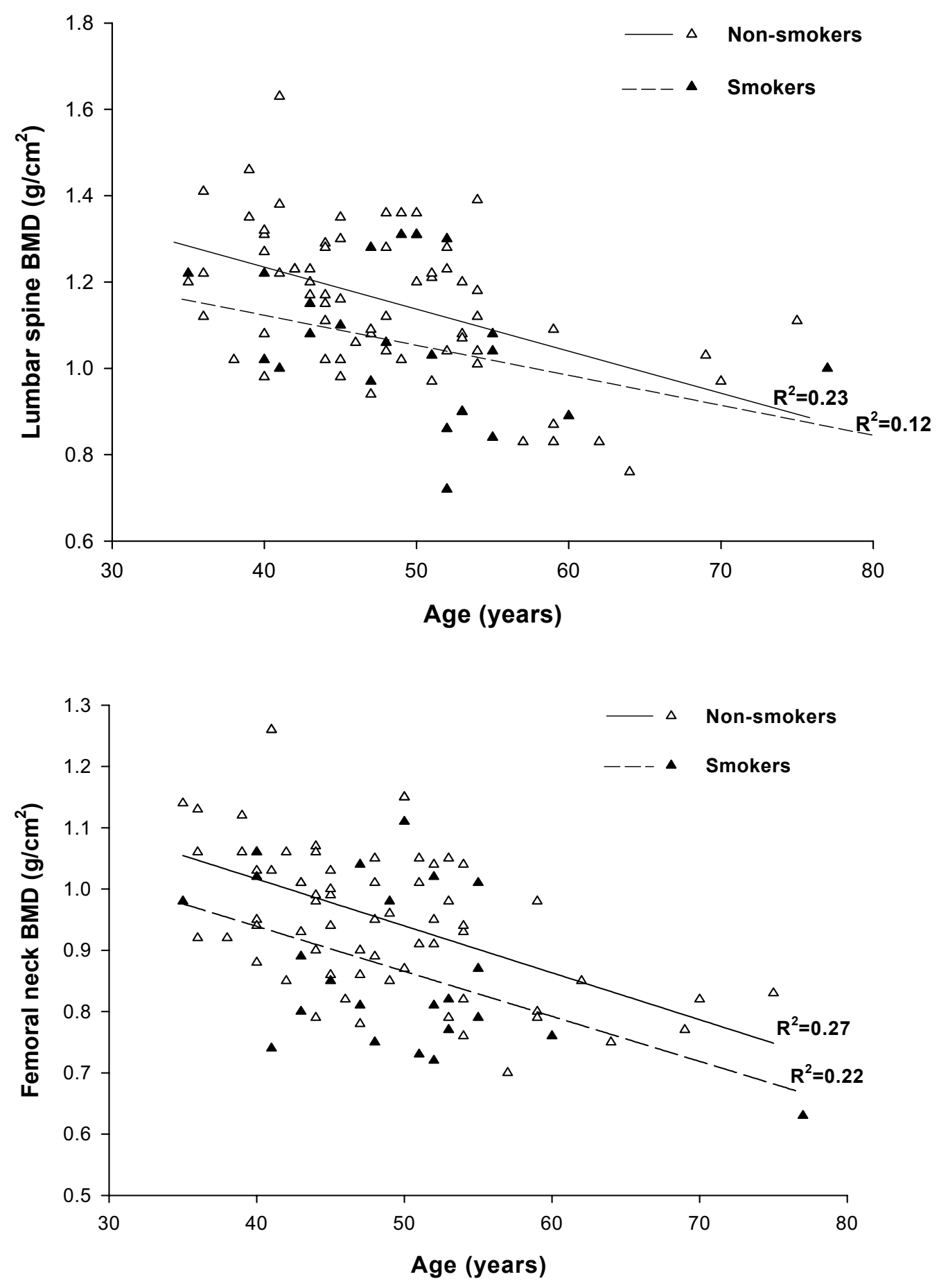

Figure 3

Interaction between age and smoking status on lumbar spine (upper panel) and femoral neck (lower panel) bone mineral density $\left(\mathrm{g} / \mathrm{cm}^{2}\right)$. 
lumbar spine and $5.3 \%(\mathrm{n}=2)$ at the femoral neck. Among smokers, the prevalence was 30.4\% (7/23) which was significantly higher $(p<0.01)$ than that among nonsmokers $(7.5 \%, 5 / 67)$.

\section{Discussion}

Osteoporosis is recognized as a public health problem in the world, but its epidemiology in non-Western populations remains poorly understood. This study represents an original contribution to the study of osteoporosis in Iranian women in Australia. Apart from advancing age and body mass index, it was found that cigarette smoking was an important modifiable determinant of bone mineral density.

These results are consistent with previous studies which indicated that advancing age was associated with lower BMD. In this study, each year increase in age was estimated to cross-sectionally "decrease" $0.8 \%$ in LS and FN BMD. This estimate is relatively consistent with longitudinal studies in Western Caucasian women which suggest an annual decrease of $1 \%[22,23]$.

Consistent with previous studies[8,9], this study found that lower BMI was associated with lower BMD. Moreover, the effect of smoking was significant in obese women. The prevalence of current cigarette smoking in this population $(17.8 \%)$ was surprisingly higher than Iranian women in Iran which was around 3.6\%[24]. BMD in smokers was $8 \%$ lower than in non-smokers, after adjusting for age and BMI. This difference is clinically significant, because each SD reduction in BMD is associated with a 2-fold increase in age adjusted fracture risk.

BMD measurements in ex-smokers were intermediate between current and non-smokers. It seems the effect of smoking varies linearly with the intake of cigarette and may suggest that smoking cessation have a positive effect on both LS and FN BMD. This makes cigarette smoking one of the important modifiable lifestyle risk factors of osteoporosis in Iranian Australian women.

Although some studies found an association between calcium intake and BMD, the present study found no such association. The results also revealed a non-significant relationship between exercise and BMD. One of the reasons for these results may be that the questionnaire only reflected the present situation, not permanent lifestyle. In addition, frequency and type of exercise on bone density could not be evaluated, because few of the subjects exercised regularly.

Alcohol use was not significantly associated with bone mineral density. Although cigarette smoking was common in this sample, alcohol use was not frequent and only a few (11\%) women reported drinking any kind of alcohol, with the majority drinking only monthly or rarely. With such a low prevalence of use, it is perhaps not surprising that the study was unable to detect a significant effect of alcohol use on BMD.

Using T-scores from two bone sites the prevalence of osteoporosis (T-scores $\leq-2.5)$ was $3.8 \%(n=2)$ and $26.3 \%(n$ $=10$ ) in pre-and post-menopausal women, respectively. The most notable observation in this study is that osteoporosis was more likely to be identified at the lumbar spine than the femoral neck. This finding is consistent with a previous study among Iranian women in Iran [25]. However, the prevalence of osteoporosis at the femoral neck seemed to be relatively lower in the Iranian women compared with most Asian and other Caucasian populations [26-29]. These results suggest that BMD measured at the femoral neck requires further investigation in Iranian women.

The present study's findings must be interpreted within the context of a number of strengths and weaknesses. This study is the first attempt to address an important public health problem amongst Iranian women in an Australian setting. Some aspects of acculturation should be taken into account in the interpretation of these findings. Since $75 \%$ of women have settled in Australia for at least 5 years, change of lifestyle factors such as sun exposure and diet cannot be ruled out, and the present results may not be generalizable to Iranian women in Iran. The association between risk factors and BMD as observed in this study cannot be interpreted as a causal relationship because the study was a cross-sectional investigation. Because the women in this study were not sampled from an age-stratified scheme, the average T-scores and prevalence of osteoporosis could have been affected by the actual age group distribution, and this represents a potential limitation for generalizing the results to the general population.

\section{Conclusion}

These data suggest that, apart from advancing age and lower BMI, cigarette smoking is an important modifiable determinant of bone mineral density in the Iranian Australian women. These findings can potentially contribute toward the development of more effective public health strategies for the health promotion and osteoporosis prevention in this population.

Further studies are required to investigate the effect of changing environmental exposures which can influence osteoporosis prevalence and fracture risk in this population. 


\section{Competing interests}

The author(s) declare that they have no competing interests.

\section{Authors' contributions \\ Conception and study design: AB, JE, TN}

Data collection: AB, NP

Drafting manuscript: $\mathrm{AB}$

Data analysis: $\mathrm{AB}, \mathrm{NN}, \mathrm{TN}$

Review of manuscript for important intellectual content: $\mathrm{AB}, \mathrm{NP}, \mathrm{JE}, \mathrm{TN}$

All authors read and approved the final manuscript.

\section{Acknowledgements}

The Iranian Ministry of Health and Medical Education is thanked for the award of a scholarship to $A B$. The authors acknowledge the assistance of Ms Fiona McGrath in the measurement of bone densitometry. We wish to express our thanks to A/Prof Jan Ritchie for her comments. We would like to thank Mr. Hamid Atighpour for his assistance with the data collection. We also thank the Iranian women for their participation in this study.

\section{References}

I. Melton LJ 3rd: Adverse outcomes of osteoporotic fractures in the general population. J Bone Miner Res 2003, I 8: I I39- I | 4 I.

2. Hansen LB, Vondracek SF: Prevention and treatment of nonpostmenopausal osteoporosis. Am J Health Syst Pharm 2004, 6 I:2637-54.

3. Cummings SR, Melton LJ: Epidemiology and outcomes of osteoporotic fractures. Lancet 2002, 359:176I-67.

4. Randell A, Sambrook PN, Nguyen TV, Lapsley H, Jones G, Kelly PJ, Eisman JA: Direct clinical and welfare costs of osteoporotic fractures in elderly men and women. Osteoporos Int 1995, 5:427-432.

5. Marshall D, Johnell O, Wedel H: Meta-analysis of how well measures of bone mineral density predict occurrence of osteoporotic fractures. BM] I996, 3 | 2: | 254-59.

6. Melton LJ, Atkinson EJ, O'Fallon WM, Wahner HW, Riggs BL: Long term fracture risk prediction by bone mineral density assessed at different skeletal sites. J Bone Miner Res 1993, 8: $1227-33$.

7. Nguyen TV, Sambrook PN, Kelly PJ, Jones G, Lord S, Freund J, Eisman J: Prediction of osteoporotic fractures by postural instability and bone density. BMJ 1993, 307:IIII-I5.

8. Felson DT, Zhang Y, Hannan MT, Anderson J): Effects of weight and body mass index on bone mineral density in men and women: the Framingham study. J Bone Miner Res 1993, 8:567-73.

9. Nguyen TV, Center JR, Eisman JA: Osteoporosis in elderly men and women: effects of dietary calcium, physical activity, and body mass index. J Bone Miner Res 2000, I 5:322-31.

10. Nguyen TV, Center JR, Eisman JA: Osteoporosis: underrated, underdiagnosed and undertreated. Med J Aust 2004, I 80(5 Suppl):S18-22.

II. Gerdhem P, Obrant KJ: Effects of cigarette smoking on bone mass as assessed by dual-energy $X$-ray absorptiometry and ultrasound. Osteoporos Int 2002, I3:932-36.

12. Ward KD, Klesges RC: A meta-analysis of the effects of cigarette smoking on bone mineral density. Calcif Tissue Int 200I, 68:259-70.

13. Jones G, Scott FS: A cross-sectional study of smoking and bone mineral density in premenopausal parous women: effect of body mass index, breastfeeding, and sports participation. J Bone Miner Res 1999, I4:1628-633.

14. Maddah M, Eshraghian MR, Djazayery A, Mirdamadi R: Association of body mass index with educational level in Iranian men and women. Eur J Clin Nutr 2003, 57:819-23.

15. Mirmiran P, Mohammadi F, Sarbazi N, Allahverdian S, Azizi F: Gender differences in dietary intakes, anthropometrical measurements and biochemical indices in an urban adult population: the Tehran Lipid and Glucose Study. Nutr Metab Cardiovasc Dis 2003, | 3:64-7|

16. Doheny M, Sedlak C: Osteoporosis Preventive Behavior Survey, Kent State University, 19952000.

17. National Food Authority: The Supplement to NOTTAB95M. Canberra: Australian Government Publishing Service; 1995.

18. World Health Organization: Preventing and managing the global epidemic: report of the WHO consultation on obesity. Geneva, WHO; 1998.

19. World Health Organization: Assessment of fracture risk and its application to screening for postmenopausal osteoporosis. Report 843. Geneva: World Health Organization; 1994.

20. SAS Institute: SAS/STAT: User's Guides. SAS Institute, Cary, NC; 1990.

21. SPSS Inc: SPSS for Windows, Release I I.0.0. Chicago: IL: SPSS, Inc; 2001.

22. Jones G, Nguyen T, Sambrook P, Kelly PJ, Eisman JA: Progressive loss of bone in the femoral neck in elderly people: longitudinal findings from the Dubbo osteoporosis epidemiology study. BMJ 1994, I 7:309(6956):691-695.

23. Nguyen TV, Sambrook PN, Eisman JA: Bone loss, physical activity, and weight change in elderly women: the Dubbo Osteoporosis Epidemiology Study. J Bone Miner Res 1998, I3:1458-67.

24. Ahmadi J, Khalili H, Jooybar R, Namazi N, Mohammadagaei P: Prevalence of cigarette smoking in Iran. Psychol Rep 200I, 89:339-4I.

25. Rassouli A, Milanian I, Moslemi-Zadeh M: Determination of serum 25-hydroxyvitamin D (3) levels in early postmenopausal Iranian women: relationship with bone mineral density. Bone 200I, 29:428-30.

26. Looker AC, Orwoll ES, Johnston CC Jr, Lindsay RL, Wahner HW, Dunn WL, Calvo MS, Harris TB, Heyse SP: Prevalence of low femoral bone density in older U.S. adults from NHANES III. J Bone Miner Res 1997, I 2:1761-8.

27. Iki M, Kagamimori S, Kagawa Y, Matsuzaki T, Yoneshima H, Marumo $\mathrm{F}$ : Bone mineral density of the spine, hip and distal forearm in representative samples of the Japanese female population: Japanese Population-Based Osteoporosis (JPOS) Study. Osteoporos Int 200I, I 2:529-37.

28. Wu XP, Liao EY, Huang G, Dai RC, Zhang H: A comparison study of the reference curves of bone mineral density at different skeletal sites in native Chinese, Japanese, and American Caucasian women. Calcif Tissue Int 2003, 73: I 22-32.

29. Limpaphayom KK, Taechakraichana N, Jaisamrarn U, Bunyavejchevin S, Chaikittisilpa S, Poshyachinda M, Taechamahachai C, Havanond P, Onthuam Y, Lumbiganon P, Kamolratanakul P: Prevalence of osteopenia and osteoporosis in Thai women. Menopause 200I, 8:65-9.

\section{Pre-publication history}

The pre-publication history for this paper can be accessed here:

http://www.biomedcentral.com/1471-2474/6/34/prepub 